- Case Report

\title{
An Uncommon Side Effect of a Commonly Used Antibiotic: Amoxicillin- Clavulanic Acid Induced Hepatitis
}

\author{
Vinay Mathew Thomas', Neena Thomas-Eapen ${ }^{2 * *}$ \\ ${ }^{1}$ Medical College, Thiruvananthapuram, Kerala, India \\ ${ }^{2}$ Department of Family and Community Medicine, University of Kentucky, Lexington, KY, USA
}

Amoxicillin-Clavulanic acid continues to be one of the most commonly used antibiotic combinations. Hepatic injury due to this antibiotic is rare. We report a case of amoxicillin-clavulanic acid induced hepatitis causing painless jaundice to bring to attention this rare side effect of this commonly used antibiotic. This is a case of a 62-year-old Caucasian female, who presented with acute onset severe painless jaundice, nausea, vomiting, and pruritus of less than 1-week duration. She had completed a course of amoxicillin-clavulanic acid 3 weeks prior to presentation. A careful history pointed to this simple diagnosis. It may be easily missed without an in-depth history and the patient may be subjected to unnecessary expensive tests. This case is reported to highlight cost conscious care by keeping in mind a rare side effect of the commonly used antibiotic.

Keywords: Amoxicillin-Clavulanic Acid; Hepatitis; Hepatology; Drug Reactions; Jaundice; Pruritus 


\section{INTRODUCTION}

Amoxicillin is a semi synthetic antibiotic, which was combined with clavulanic acid, to confer activity against beta lactamase produced by bacteria, thus extending its spectrum of activity to include many gram negative and positive bacteria. Marketed for the first time in $1981,{ }^{1)}$ the amoxicillin-clavulanic acid combination ushered in a new era of antibiotics. It still continues to be one of the most commonly used antibiotics and is commonly used to treat sinus, respiratory tract, and skin infections. According to a report by the Centers for Disease Control and Prevention, there were 23.1 million outpatient prescriptions of amoxicillin-clavulanic acid in United States in 2013, the third most commonly prescribed antibiotic after amoxicillin and azithromycin. ${ }^{2)}$

Although usually well tolerated, there are a number of side effects associated with this drug. The addition of clavulanic acid to amoxicillin not only changes the antimicrobial activity, but also the spectrum of side effects, as described in a study by Salvo et al. ${ }^{3)}$ They analyzed the frequency of different side effects in six Italian regions from 1988 to 2005. They found that the frequency of skin reactions was significantly higher for amoxicillin (82\%) than for amoxicillin-clavulanic acid (76\%); on the contrary, the frequency of gastrointestinal, hepatic, and hematological reactions was significantly higher for amoxicillin-clavulanic acid ( $13 \%, 4 \%$, and $2 \%$, respectively) than for amoxicillin $(7 \%, 1 \%$, and $1 \%$, respectively). ${ }^{3)}$

Amoxicillin-clavulanic acid related hepatic injury, although rare, is well known. A retrospective cohort study done in the United Kingdom showed that the incidence of acute liver injury associated with the combination of amoxicillin-clavulanic acid and amoxicillin alone was 1.7 and 0.3 per 10,000 prescriptions, respectively. ${ }^{4)}$ There have been several case reports of amoxicillin-clavulanic acid related hepatic injury. The first case of hepatotoxicity caused by this drug was reported in 1988. ${ }^{5)}$ The hepatic injury usually shows a cholestatic pattern predominantly and, sometimes, hepatocellular and granulomatous patterns. ${ }^{6}$

This case had a clear correlation of the patient's hepatitis causing painless jaundice to her treatment with amoxicillin-clavulanic acid. The intention of reporting this case is to bring to the attention of physicians and dentists in all specialties, particularly in primary care, this rare side effect of the commonly used antibiotic.

The second reason is to highlight that the presentation can be very acute and dramatic after several weeks of stopping the antibiotic use to the extent that a clinician may consider an obstructive or neoplastic cause as the first differential diagnosis. Only a careful history with this complication in mind will avoid unnecessary invasive work-up.

\section{CASE REPORT}

A 62-year old Caucasian female, a retired nurse with known rheumatoid arthritis, presented to the clinic for acute onset severe jaundice, nausea, vomiting, and pruritus of less than 1-week duration without any other constitutional or systemic symptoms. The patient was not currently on any disease modifying medications for rheumatoid arthritis for the past 8 months. Her regular medications included loratidine for allergic symptoms, multivitamin and vitamin D as supplements, tramadol as necessary for joint pain, and primidone for essential tremors. She also did not have any history of liver disease and was not an alcoholic.

On physical examination, her vital signs were normal and stable. Scleral conjunctiva showed visible severe jaundice. Skin showed markings from intense generalized pruritus. She had also noticed a fine rash on her skin, which resolved spontaneously. Abdominal examination was normal without any hepatosplenomegaly, or signs of liver failure or portal hypertension. There were no abdominal masses felt on palpation.

In-depth exploration of the history revealed that the patient had undergone dental work 3-4 weeks prior and was given a 10-day course of amoxicillin-clavulanic acid, which she completed 3 weeks prior to presentation. She had no symptoms or signs until 1 week prior to the presentation to the clinic. She had no previous history of amoxicillinclavulanic acid related reactions, but had allergy in the form of skin eruptions to other antibiotics like sulfa drugs, nitrofurantoin, penicillin, gold salts, and adalimumab.

Laboratory tests showed markedly elevated liver transaminases, alkaline phosphatase, and elevated total bilirubin with normal glucose, electrolytes, and blood counts. Viral hepatitis panel was negative. This included acute hepatitis panel, which included hepatitis C antibody, hepatitis B surface antigen and core antibodies immunoglobulin M (IgM) and immunoglobulin (IgG), and hepatitis A IgM and IgG antibodies. Ultrasound of the liver and gall bladder did not show any specific pathology to explain the jaundice and abnormal liver functions.

The patient was seen by a gastroenterologist. The gastroenterologist ordered a magnetic resonance cholangio-pancreatogram, after initial evaluation. It showed a normal biliary system and no extra or intrahepatic biliary duct dilatation.

The gastroenterologist concluded that this was amoxicillin-clavu-

Table 1. Liver function tests over the course of treatment and follow-up

\begin{tabular}{|c|c|c|c|c|c|c|}
\hline Variable & Reference range & October 19 & October 22 & October 29 & November 9 & February 9 \\
\hline Aspartate aminotransferase (IU/L) & $8-36$ & 235 & 585 & 148 & 30 & 34 \\
\hline Alanine aminotransferase (IU/L) & 8-33 & 231 & 425 & 259 & 40 & 41 \\
\hline Alkaline phosphatase (IU/L) & $46-142$ & 677 & 1,083 & 1,046 & 504 & 220 \\
\hline Total bilirubin (mg/dL) & $0.2-1.1$ & 6.8 & 11 & 5 & 2 & 0.3 \\
\hline Conjugated bilirubin (mg/dL) & $0-0.2$ & 5.3 & 8.7 & 2.7 & & \\
\hline Albumin $(\mathrm{g} / \mathrm{dL})$ & $3.3-4.6$ & 3.1 & 2.8 & 3 & 3.3 & 3.6 \\
\hline
\end{tabular}


lanic acid induced hepatitis and no further investigation was required. The patient and all physicians involved mutually agreed to wait and watch the liver functions over a course of several weeks. The only medication given to the patient was cholestyramine for the pruritis.

Serial measurement of her liver functions over a course of 3 months showed complete resolution of the elevated transaminases and bilirubin (Table 1, Figures 1,2). The patient clinically improved and made a complete recovery.

\section{DISCUSSION}

The first case of amoxicillin-clavulanic acid induced hepatic injury was reported in 1988 in the Netherlands. ${ }^{5}$ There have been several such case reports since then. Although rare, this is a well-known complication. There have been cases of hepatic injury caused by amoxicillin alone; however, the risk is more with the amoxicillin-clavulanic acid combination. ${ }^{3)}$

Since this side effect is not seen in everyone consuming this antibiotic, several risk factors have been postulated. Studies have identified that hepatotoxicity is more common in males, ${ }^{6}$ leading to suggestions that there is a sexual influence in amoxicillin-clavulanic acid induced hepatotoxicity. ${ }^{6)}$ This side effect has also been seen more commonly in older age groups. ${ }^{7)}$ However, there have been some case reports of amoxicillin-clavulanic acid induced hepatotoxicity in young patients as well. The duration of treatment has also been cited as a risk factor in some reports. ${ }^{6,7)}$

The pathogenesis of amoxicillin-clavulanic acid induced hepatitis has not been fully understood. There have been postulates that idiosyncratic immune-allergic reactions are the cause. ${ }^{7-9)}$ This postulate is based on the fact that hepatotoxicity coexists with hypersensitivity reactions such as skin rashes and hypereosinophilia, with the presence of specific antibodies like anti-mitochondrial type 6, anti-liver/kidney microsome 2, and anti-liver microsomal antibodies, ${ }^{9)}$ and liver biopsy

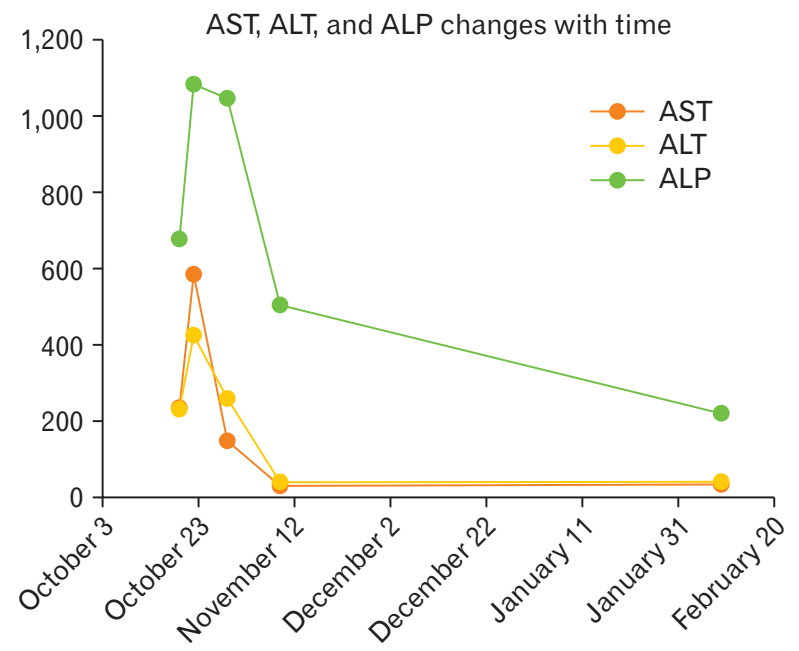

Figure 1. Levels of AST, ALT, and ALP over 3 months (IU/L). AST, aspartate aminotransferase; ALT, alanine aminotransferase; ALP, alkaline phosphatase. showing eosinophilic infiltrates. ${ }^{9)}$ Genetic associations with human leukocyte antigen class II antigens such as DRB1*1501-DRB5*0101DQB1*0602 haplotype have also been found. ${ }^{1)}$

Our patient presented with cholestatic features like pruritus, jaundice, and elevated liver enzymes. The most common pattern of hepatic injury described has been of the cholestatic type. Mixed, hepatocellular, and granulomatous patterns may also occur. The hepatotoxicity usually follows a temporal pattern, and usually occurs 1-8 weeks after the use of the antibiotic ${ }^{9)}$ and it followed the same temporal pattern in our patient. To improve the diagnosis of suspected drug induced liver injury, instruments to assess causality have been introduced. ${ }^{10)}$ There is no gold standard laboratory or diagnostic test for drug induced liver injury, and thus, these instruments are validated by comparing results to those of an experienced expert panel. ${ }^{9)}$ One of the scoring systems is Council for International Organizations of Medical Sciences (CIOMS) scale. The CIOMS score for our patient was 9 , which showed that drug induced liver injury was highly probable.

Liver biopsies have been used to diagnose it, as mentioned in many of the case reports, and the typical findings associated with it include peri-portal lymphocytic infiltration and hepatocyte damage. ${ }^{8,9)}$ A liver biopsy was not done in this case, since other causes were ruled out and amoxicillin-clavulanic acid induced hepatotoxicity was highly suspected. This is an important message. If amoxicillin-clavulanic acid induced hepatotoxicity is suspected and other serious causes have been ruled out, watchful waiting might be prudent. As long as the downward trend of hepatic enzymes is noticed, a patient may be closely followed until the liver functions revert to normal. This would save the patient from painful procedures like liver biopsy and help in bringing down the treatment costs.

Amoxicillin-clavulanic acid induced hepatotoxicity generally follows a benign course and has a good prognosis, although reports of fulminant hepatitis resulting in death have also been reported. ${ }^{9)}$ Symptomatic treatment is all that is usually required. Since the mechanism

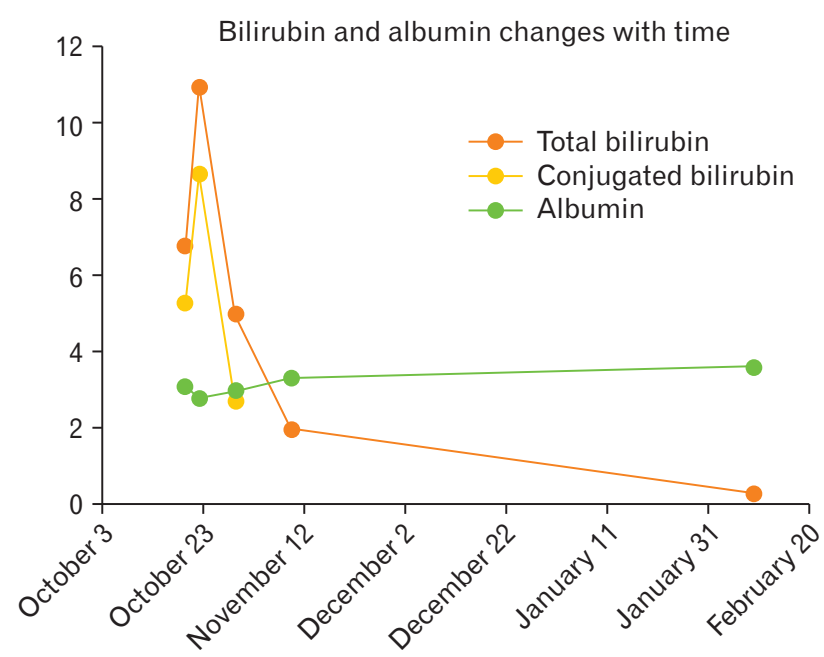

Figure 2. Serum bilirubin ( $\mathrm{mg} / \mathrm{dL})$ and albumin $(\mathrm{g} / \mathrm{dL})$ over 3 months. 
of hepatic injury has been postulated to be inflammatory, some have suggested treatment with corticosteroids, ${ }^{8)}$ but no clear reduction in morbidity has been established.

This patient should not be prescribed amoxicillin-clavulanic acid again, since studies have shown that re-challenge with the drug can result in the same injury. ${ }^{6)}$

The learning point from this case is to be aware of this rare side effect of the commonly prescribed antibiotic. It usually occurs 3-6 weeks after completion of the therapy. Unless a very careful and thorough history going back to a few months is taken, this simple diagnosis may be missed in a case of acute painless jaundice and the patient might be subjected to unnecessary expensive tests. Due to heightened awareness, if this differential diagnosis is considered and established in a case of painless jaundice, with gradual stepwise work-up, the patient can be reassured about the disease process. Careful watchful waiting with close monitoring of liver functions may be the only follow-up needed. This type of cost conscious care is valuable in this era of valuebased care.

\section{CONFLICT OF INTEREST}

No potential conflict of interest relevant to this article was reported.

\section{REFERENCES}

1. O'Donohue J, Oien KA, Donaldson P, Underhill J, Clare M, MacSween RN, et al. Co-amoxiclav jaundice: clinical and histological features and
HLA class II association. Gut 2000;47:717-20.

2. Centers for Disease Control and Prevention. Outpatient antibiotic prescriptions-United States, 2013 [Internet]. Atlanta (GA): Centers for Disease Control and Prevention; 2013 [cited 2016 Aug 20]. Available from: http://www.cdc.gov/getsmart/community/pdfs/annual-reportsummary_2013.pdf.

3. Salvo F, Polimeni G, Moretti U, Conforti A, Leone R, Leoni O, et al. Adverse drug reactions related to amoxicillin alone and in association with clavulanic acid: data from spontaneous reporting in Italy. J Antimicrob Chemother 2007;60:121-6.

4. Garcia Rodriguez LA, Stricker BH, Zimmerman HJ. Risk of acute liver injury associated with the combination of amoxicillin and clavulanic acid. Arch Intern Med 1996;156:1327-32.

5. Van den Broek JW, Buennemeyer BL, Stricker BH. Cholestatic hepatitis caused by a combination of amoxicillin and clavulanic acid (Augmentin). Ned Tijdschr Geneeskd 1988;132:1495-7.

6. Larrey D, Vial T, Micaleff A, Babany G, Morichau-Beauchant M, Michel $\mathrm{H}$, et al. Hepatitis associated with amoxycillin-clavulanic acid combination report of 15 cases. Gut 1992;33:368-71.

7. Zaidi SA. Hepatitis associated with amoxicillin/clavulanic acid and/or ciprofloxacin. Am J Med Sci 2003;325:31-3.

8. Herrero-Herrero JI, Garcia-Aparicio J. Corticosteroid therapy in a case of severe cholestasic hepatitis associated with amoxicillin-clavulanate. J Med Toxicol 2010;6:420-3.

9. Fontana RJ, Shakil AO, Greenson JK, Boyd I, Lee WM. Acute liver failure due to amoxicillin and amoxicillin/clavulanate. Dig Dis Sci 2005;50:1785-90.

10. Benichou C. Criteria of drug-induced liver disorders: report of an international consensus meeting. J Hepatol 1990;11:272-6. 